\section{Indicadores de circulación: una perspectiva multi-escalar para medir la producción científico- tecnológica latinoamericana}

\begin{abstract}
Resumen: En el concepto de que existe una ciencia de corriente principal y, por oposición, una ciencia "periférica", las publicaciones en revistas indexadas por Web of Science y el tipo de indicadores que se usan para medir la producción científico tecnológica (CT) mundial, han jugado, sin duda, un rol fundamental, ya que pasaron a ser el eje principal de la evaluación institucional e individual también en la periferia. De ahí la necesidad urgente de crear nuevas herramientas de medición de la producción CT en la periferia, que puedan romper con el círculo vicioso que mercantiliza las culturas evaluativas. Los indicadores de circulación se han propuesto como una respuesta posible a este desafío, ya que contemplan la diversidad de estilos de producción y la multi-escalaridad de las interacciones de las universidades. En este trabajo se presentan avances realizados en esta materia. Por un lado, en relación con el circuito latinoamericano de revistas científicas, que no ha logrado ofrecer aún indicadores regionales de la producción CT publicada, para que tenga relevancia en los procesos de evaluación. Por otro lado, en relación con los estilos de producción de conocimiento en las universidades, para poder examinar la circulación de la producción CT en una escala local. Palabras claves: Indicadores de circulación, multi-escalaridad, circuitos de reconocimiento, estilos institucionales, producción de conocimiento.
\end{abstract}

Circulation indicators: a multi-scalar perspective to measure the Latin American scientific and technological production

\section{Abstract}

In a scenary characterized by a mainstream science and, by contrast, a "peripheral" science, publications in journals indexed by Web of Science and the type of indicators used to measure world scientific and technological (ST) production, have played, without doubt, a fundamental role as they became the main elements used for the institutional and individual evaluation also in the periphery. Hence the urgent need to create new tools for measuring the periphery ST production suitable to break the vicious circle that commercializes evaluative cultures. Circulation indicators have been proposed as a possible response to this challenge, since they contemplate the diversity of production styles and the multi-scalarity of university interactions. This paper presents advances made in this area. On the one hand, in relation to the Latin American circuit of scientific journals, which has not yet been able to offer regional indicators of the published ST production for its valorization in evaluation processes. On the other hand, in relation to the styles of knowledge production in universities, in order to examine the circulation of ST production on a local scale.

Keywords: Circulation indicators, multi-scalarity, recognition circuits, institutional styles, knowledge production.

Indicadores de circulação: uma perspectiva multi-escalar para medir a produção científico-tecnológica latino-americana

Resumo: Tendo por base a conceitualização de dois tipos de ciências -uma dominantee, em oposição, uma "periférica"-, as publicações em periódicos indexados pela Web of Science e o tipo de indicadores usados para medir a produção científica tecnológica global (CT) têm desempenhado, sem dúvida, um papel fundamental, já que se tornaram o eixo principal de avaliação institucional e individual também na periferia. Daí a necessidade urgente de criar novas ferramentas para medir a produção de CT na periferia, o que possam romper o círculo vicioso que mercantiliza as culturas avaliativas. Os indicadores de circulação têm sido propostos como uma possível resposta a esse desafio, já que contemplam a diversidade de estilos de produção e a natureza multi-escalar das interações universitárias Este artigo apresenta os avanços nessa área. Por um lado, em relação ao circuito latino-americano de revistas científicas, que ainda não foi capaz de oferecer indicadores regionais da produção publicada de CT, para que tenha relevância nos processos de avaliação; por outro lado, em relação aos estilos de produção de conhecimento nas universidades, para poder examinar a circulação da produção de CT em escala local.

Palavras chaves: Indicadores de circulação, multi-escalaridade, circuitos de reconhecimento, estilos institucionais, produção de conhecimento.

\section{Ciencia Tecnología y Política \\ Año 2 Nº 3 jul- dic 2019}

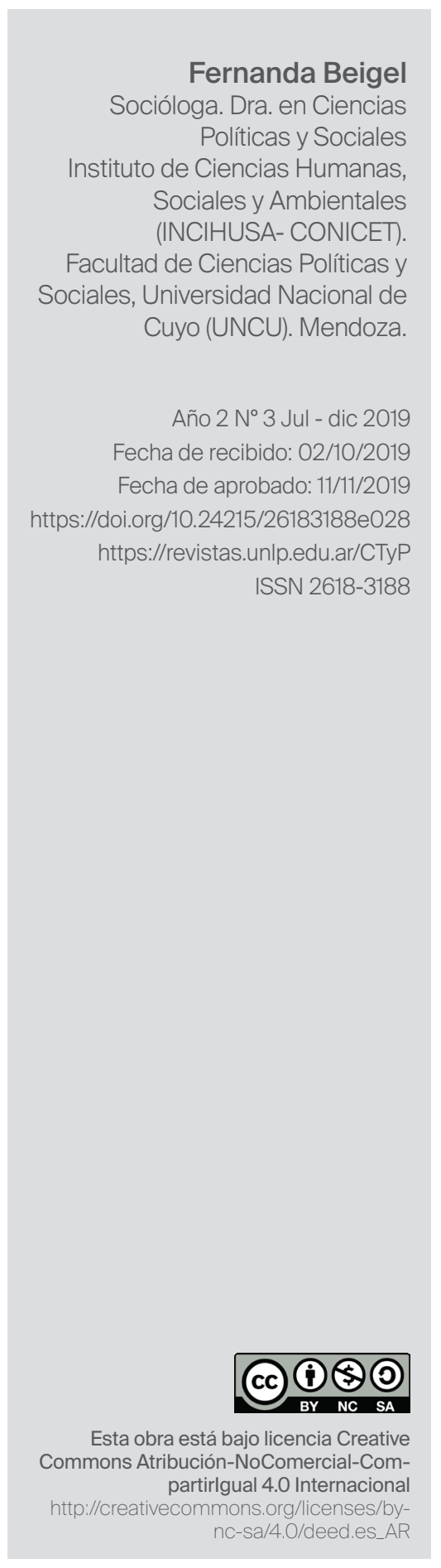

UNIVERSIDAD

NACIONAL

DE LA PLATA 


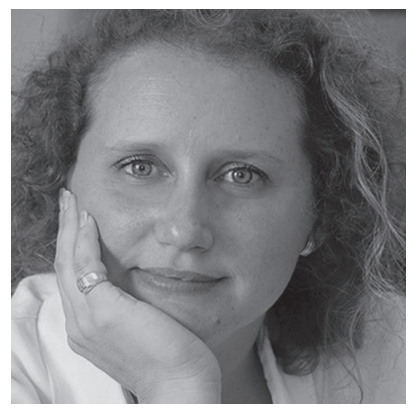

\section{Fernanda Beigel}

Socióloga. Dra. en Ciencias

Políticas y Sociales

Instituto de Ciencias Humanas,

Sociales y Ambientales (INCl-

HUSA- CONICET).

Facultad de Ciencias Políticas y

Sociales, Universidad Nacional

de Cuyo (UNCU). Mendoza.

\section{Indicadores de circulación: una perspectiva multi-escalar para medir la producción} científico-tecnológica latinoamericana

Resumen: En el concepto de que existe una ciencia de corriente principal y, por oposición, una ciencia "periférica", las publicaciones en revistas indexadas por Web of Science y el tipo de indicadores que se usan para medir la producción científico tecnológica (CT) mundial, han jugado, sin duda, un rol fundamental, ya que pasarona ser eleje principal de la evaluación institucional e individual también en la periferia. De ahí la necesidad urgente de crear nuevas herramientas de medición de la producción CT en la periferia, que puedan romper con el círculo vicioso que mercantiliza las culturas evaluativas. Los indicadores de circulación se han propuesto como una respuesta posible a este desafío, ya que contemplan la diversidad de estilos de producción y la multi-escalaridad de las interacciones de las universidades. En este trabajo se presentan avances realizados en esta materia. Por un lado, en relación con el circuito latinoamericano de revistas científicas, que no ha logrado ofrecer aún indicadores regionales de la producción CT publicada, para que tenga relevancia en los procesos de evaluación. Por otro lado, en relación con los estilos de producción de conocimiento en las universidades, para poder examinar la circulación de la producción CT en una escala local.

\section{Introducción}

Existen numerosos estudios sobre las relaciones de poder que atraviesan la producción del conocimiento científico y el modo en que se fue construyendo el concepto de ciencia "mainstream" y, por oposición, la idea de ciencia "periférica", incluyendo en esta última todo lo que quedaba afuera de la producción registrada en las revistas indexadas por Web of Science (Vessuri, 1995; Guédon, 2011). Aunque existen muy diversos niveles de producción de conocimientos científicos en la llamada "periferia" y ciertos países han alcanzado el nivel de "centros periféricos", buena parte de las dificultades para conocer sus especificidades reside en el tipo de indicadores que se usan para medir la producción científica mundial y establecer comparaciones. Arvanitis y Gaillard (1992) argumentaron tempranamente que era esencial considerar la "especificidad de la cienciometría de los países en desarrollo" puesto que, más que una diferencia de calidad con los países centrales, la distancia era cuestión de proporciones. Schott (1988) planteó que los 
centros académicos tradicionales habían sido muy eficientes en acumular "centralidad", algo que era observable en la cantidad de lazos que unían, unidireccionalmente, las periferias con los centros en las colaboraciones internacionales. Al mismo tiempo determinó que esa dominación estaba muy por encima de la verdadera performance de esos centros.

Estas constataciones contribuyeron a desmitificar la productividad de los centros y señalaron los sesgos de los indicadores tradicionales, pero no cuestionaron en sí las raíces de las que se nutre la dicotomía "mainstream/periferia", que están justamente en las bases de datos estructuralmente limitadas que han producido y reproducido esa centralidad. Gracias al monopolio que el Institute for Scientific Information (ISI) tuvo de los sistemas de indexación e indicadores bibliométricos, durante más de cuarenta años, ciertas instituciones, disciplinas y lenguas acumularon capital científico medible en citaciones, mientras despojaban a otras áreas, disciplinas y lenguas del prestigio científico que confería participar en las revistas de corriente principal. En otros estudios (Beigel, 2014) analizamos cómo ese sistema de publicación se convirtió en un circuito de reconocimiento por excelencia, que la "globalización académica" se encargó de reificar en la forma de "prestigio internacional" para un grupo selecto de universidades que pronto entrarían en el top ten de los rankings mundiales.

En gran medida, el concepto de ciencia de corriente principal se consolidó porque las publicaciones pasaron a ser el eje principal de la evaluación institucional e individual también en la periferia. El uso de la bibliometría contribuyó a reforzar la convicción de que el inglés funciona como lingua franca y la neutralidad de un estilo progresivamente homogéneo de escritura y publicación académico (Heilbron, 2002). La "univer- salización" de estas tendencias fue impulsada además por el creciente interés de académicos de todo el mundo por ingresar al complejo ISI-Web of Science (hoy Clarivate), lo que llevó a muchas revistas de comunidades periféricas deseosas de "pertenecer", a cambiar al idioma inglés. En otros trabajos (Beigel, 2018) se ha destacado que el éxito de la "globalización académica" no habría sido posible sin los efectos perniciosos de los rankings universitarios. Gracias a éstos el círculo vicioso de la mercantilización del sistema de publicaciones terminó encerrando a los propios profesores/as, cuando las instituciones y los gobiernos comenzaron a reorientar las culturas evaluativas para ajustarlas a la producción en los términos de la "corriente principal".

La crítica a las distorsiones generadas por el circuito mainstream y en particular al Factor de Impacto (FI) tiene una amplia tradición en el movimiento de acceso abierto latinoamericano donde han surgido iniciativas no mercantiles y modelos cooperativos gestionados por el propio sector académico. Es así que actores y expertos provenientes de repositorios regionales y redes académicas, como por ejemplo el Consejo Latinoamericano de Ciencias Sociales (CLACSO), han desarrollado un análisis profundo acerca de las relaciones entre "excelencia" y "calidad" (Babini, 2011; Cetto \& Alonso, 2011). Por otro lado en América Latina son principalmente las universidades las instituciones editoras de las revistas científicas. Éstas se producen a veces artesanalmente y con muchas dificultades, otras con mayor apoyo institucional, pero en su gran mayoría con fondos públicos: la tendencia general es al acceso abierto y la difusión en portales universitarios.

Es por ello que venimos sosteniendo la necesidad urgente de crear nuevas herramientas de medición de la producción científica de la periferia que puedan romper con el círculo vicioso 
que mercantiliza las culturas evaluativas. Y así, reinstalar al propio sector académico en el control de su sistema de comunicaciones. En esta dirección, hemos creado los indicadores de circulación que contemplan la diversidad de estilos de producción y la multi-escalaridad de las interacciones de las universidades, no sólo con los circuitos dominantes sino también con otros circuitos. Se trata de una forma de medición de la producción científica que implica una transición no sólo técnica, sino también conceptual, para superar la noción unidireccional de la "internacionalización". Estos indicadores pretenden recuperar las conexiones locales que vehiculizan el impacto social del conocimiento científico y valorizar las interacciones regionales que tienen una particular fuerza en América Latina y constituyen una forma alternativa de internacionalización (Beigel, 2018).

En este trabajo, presentamos los avances que venimos realizando en materia de indicadores de circulación en dos niveles. Primeramente, en relación con el circuito latinoamericano, que supo ser un espacio de reconocimiento académico relevante pero no ha conseguido hasta el momento ofrecer indicadores regionales sobre la producción científica publicada para valorizarla en los procesos de evaluación. En segundo lugar, en relación con los estilos de producción en las universidades, que permite examinar la circulación en una escala local.

\section{El circuito latinoamericano de consagración académica}

Mientras evolucionaba el proceso de acumulación de capital científico en los centros y se catapultaban el Fl y los rankings, en América Latina la ciencia se desarrollaba en un camino diferente pero con un impulso paralelo de internacionaliza- ción. Este espacio intelectual se vio estimulado por la tendencia a la regionalización típica de la segunda posguerra que recibió apoyo financiero de agencias públicas y privadas de los países centrales. Por lo cual existe una tendencia a suponer que el desarrollo científico y universitario alcanzado en la década de 1950-1960 representa un reflejo dependiente del desarrollo financiado por los centros. En otros trabajos (Beigel, 2010; Beigel y Sabea, 2014; Beigel, 2016) hemos planteado la necesidad de deconstruir el concepto de dependencia académica para superar las discusiones retóricas y avanzar en la observación histórico-concreta del desarrollo científico local con otras fuentes, construyendo nuevas bases de datos con información primaria. Los estudios disponibles muestran que el circuito regional emergió en el marco del latinoamericanismo, un movimiento que durante el período independentista ya cuestionó los límites nacionales, y luego alcanzó importantes niveles de profesionalización y autonomía académica.

Si buscamos una fecha de nacimiento de este espacio intelectual regional veremos que precede largamente al desarrollo regional visible en los años de 1950 a 1970. La "Carta a los Españoles Americanos", escrita por Juan Pablo Vizcardo y Guzmán y publicada en 1801, señala un punto de arranque de la reflexión independentista como narrativa común al subcontinente latinoamericano que evolucionó durante todo el siglo XIX en el fecundo terreno del periodismo de ideas. El cruce entre prensa y literatura fue el camino principal del desarrollo del campo intelectual y artístico hasta entrados los años de 1920, cuando aparecieron las redes editoriales del vanguardismo, estimuladas por el movimiento americano de la Reforma Universitaria. Una incipiente industria editorial comenzó a difundir a escala regional perspectivas locales y lecturas críticas 
de las teorías occidentales que fortalecieron especialmente las ciencias sociales y humanas, acompañando el proceso de modernización y masificación de las universidades. El Fondo de Cultura Económica, Siglo XXI y otras editoriales vinieron a consolidar este campo intelectual en plena etapa de especialización.

Más tarde llegaron los centros regionales de la talla de la CEPAL (1948), FLACSO (1957), CELADE (1957), DESAL (1960), ILPES (1961), ILADES (1965) y CLACSO (1967) que promovieron la aparición de formas específicas de consagración regional y corrientes de movilidad intra-regional. Las comisiones nacionales de UNESCO y los bibliotecarios/as jugaron un papel relevante en las iniciativas regionales para crear Centros de Documentación, difundir revistas, producir listados de material y boletines bibliográficos que estimularan la circulación del conocimiento producido en la región (Beigel, 2013). En una importante reunión regional auspiciada por UNESCO en 1964 en Puerto Rico, se analizó la situación de las revistas latinoamericanas, los métodos de evaluación necesarios para construir un listado de publicaciones de calidad y las perspectivas de las revistas de carácter regional con informes sobre algunas disciplinas como Agronomía, Biología, Física, Matemática, Geofísica, Ingeniería y Química (Salatino, 2018).

Estas iniciativas demuestran que el circuito regional no estaba sostenido exclusivamente por las ciencias sociales. Quesada (2019) analiza la relevancia de las redes regionales de ciencias agrarias en la primera mitad del siglo XX, relacionadas con los intereses panamericanos y la implementación de los programas agrarios en la década de 1940 en México, Colombia, Venezuela, Ecuador, y Chile. Abarzúa Cutroni (2017), por su parte, recoge los testimonios de la creación del Centro Regional de Matemáticas en Buenos

\section{Aires en 1958.}

Se pueden advertir tres etapas/capas del circuito latinoamericano que se desarrolló sobre esta vigorosa plataforma intelectual de por lo menos doscientos años de existencia. Aquél se fortaleció con las instituciones regionales y una diplomacia académica altamente entrenada, para luego dar lugar a un ecosistema de comunicación científica que enfrentó la aparición del circuito "mainstream" mediante la creación de sistemas de indexación propios como CLASE (1975) y PERIODICA (1978). Pero fue sin lugar a dudas la década de 1990 la que protagonizó la expansión de este sistema regional de revistas gracias al apoyo del gobierno mexicano, con la creación de LATINDEX en 1994 y del gobierno brasileño con la aparición de SciELO en 1998. Al poco tiempo llegaría REDALYC (2003) y América Latina se consolidará como la vanguardia del acceso abierto y la ciencia como bien público a nivel internacional.

Es probable que la poca atención otorgada a los circuitos académicos regionales en los estudios de la ciencia se deba al peso del enfoque nacional en la historiografía dominante en Europa y Estados Unidos. Pero aún en los estudios e informes que incluyen América Latina como región se sigue observando una limitación de fondo que sigue replicando datos de producción científica surgidos de las bases de datos de tipo "mainstream" sin considerar los circuitos alternativos. En definitiva, se sigue subvalorando la regionalización como si no fuese una forma de internacionalización.

La escasez de estudios regionales está relacionada también con dificultades técnicas. Por un lado, no hay sistemas para medir la circulación de la producción evaluada y publicada en América Latina porque los tres repositorios más relevantes están desconectados. SciELO, LATINDEX 
y REDALYC hicieron numerosos esfuerzos en el pasado, con apoyo de CLACSO, para mejorar el acceso web a sus indicadores y existieron importantes estudios sobre la producción regional (Alperin y Fischman, 2015). Sin embargo, los indicadores de producción sólo generan estudios por ahora independientes para las dos colecciones que ofrecen información al nivel del artículo (SciELO y REDALYC) mientras LATINDEX está en pleno proceso de recatalogación. Esto tiene un impacto directo en la capacidad de las revistas regionales para proporcionar datos que deriven en recompensas académicas en acreditaciones institucionales e individuales.

Como resultado, la acreditación institucional y los informes científicos se siguen apegando a las bases de datos de corriente principal y la producción publicada en revistas latinoamericanas es desvalorizada en nuestras propias universidades. ¿Qué sucedió entonces con este circuito académico colaborativo, con su lógica específica que desafiaba la "universalización" de la corriente principal, al tener dificultades para ofrecer formas de reconocimiento internacional alternativo para los/las investigadores/as de la región? El circuito tiene una posición dual: dominado dentro de las jerarquías "mainstream" pero dominante dentro del conocimiento publicado en español y portugués, particularmente en las ciencias sociales y humanidades. Comparados cuantitativamente, los artículos de autores latinoamericanos de las ciencias exactas y naturales son muchos más en las bases de datos como WoS y Scopus que los que se encuentran en SciELO. En cambio, muchos más son los artículos de ciencias sociales y humanas publicados en los repositorios regionales como SciELO, LATINDEX y REDALYC que en aquellas bases tradicionales. Esta posición dominante de las revistas latinoamericanas en el ámbito de las ciencias sociales tiene un impacto directo en el reconocimiento científico en ciertas instituciones y la conquista de posiciones de investigación en ciertas disciplinas. En este contexto las revistas no indexadas son cada vez más devaluadas (Beigel, 2014). La estructura de este mundo académico de circuitos y campos científicos se encuentra hoy en plena transformación. En la Comunidad Europea surgió una creciente preocupación por la mercantilización de la edición científica y se lanzó recientemente el Plan S, con la idea de acelerar una transición de las revistas al acceso abierto. Las primeras señales indican que se protegería a la industria editorial cobrando a los autores por publicar. En este contexto Babini (2019) brinda argumentos de por qué desde América Latina se ha reaccionado para que los costos no sean transferidos a los investigadores.

\section{Oliva, una nueva base de datos de la producción CyT latinoamericana}

La defensa del ecosistema latinoamericano forma parte del ideario compartido en AmeliCa, una iniciativa regional lanzada por REDALYC y auspiciada por CLACSO y UNESCO. Este es el objetivo principal del proyecto que estamos encarando desde la comisión de investigaciones de dicha organización, con un equipo de investigadores/ as y bibliotecarios/as que funciona con sede en la Universidad Nacional de Cuyo'. Se trata de una base de datos que hemos denominado Oliva y en su primera fase está fusionando la información a nivel de artículo proporcionada directamente por SciELO y REDALYC, con la meta de avanzar en

\footnotetext{
${ }^{1}$ El equipo de la comisión que coordino está compuesto por Horacio Degiorigi, Adrián Méndez, Maximiliano Salatino, Patricia Testa y Laura Fernández. Agradezco mucho la dedicación y compromiso que ponen en este proyecto.
} 
una siguiente etapa incorporando la producción indexada en DOAJ y LATINDEX-Catálogo 2.O.

Hasta el momento los artículos incorporados en la base de datos Oliva son 3.935.204 y la información de cada uno incluye la afiliación institucional y país de los/as 8.082.369 autores/as de esos artículos, los metadatos, la disciplina, resúmenes y lengua de publicación. El objetivo concreto del proyecto es producir una serie de indicadores estadísticos básicos capaces de nutrir los informes de ciencia y tecnología que se producen a nivel iberoamericano. Se propone así, dar cuenta de la producción latinoamericana evaluada que circula en acceso abierto y tiene los mismos niveles de calidad que la producción indexada en el circuito mainstream, pero que hasta el momento no ha logrado niveles de visibilidad suficientes como para incidir en las políticas evaluativas de nuestros países.

Se espera que la generación de indicadores regionales de la producción publicada en América Latina que se han incluido en esta primera etapa en la base de datos, permita modificar la incidencia de estos repositorios en los sistemas nacionales de evaluación, puesto que hasta el momento Scopus y/o Web of Science (Clarivate) siguen siendo el patrón de medida de las trayectorias para el ingreso a la carrera del investigador en países como Argentina, los concursos docentes en las Universidades y la promoción en los cargos, en la mayoría de los países de la región. Según los estudios disponibles (Alperin y Rozemblum, 2018; Vasen, 2018) en Chile, Colombia o México se observa una consolidación del modelo de publicación mainstream que ha sido promovido con sistemas de incentivo salarial para orientar a los investigadores a las revistas de "alto impacto". En contraste, Argentina ha desarrollado una posición más crítica respecto de estos indicadores y una mayor diversidad en términos de "valuación" del circuito regional. Si bien en estudios anteriores (Beigel, 2014) pudimos demostrar que por ejemplo es una práctica extendida en el caso del CONICET de Argentina, el reemplazo de la evaluación de la originalidad de las publicación por la valoración de la indexación de las revistas, en el área de ciencias sociales y humanas los repositorios regionales como SciELO y LATINDEX Catálogo, son valorados en igual nivel que WoS o SCOPUS². Esto ha reforzado una trayectoria de internacionalización de signo latinoamericanista en estos investigadores/as que se distinguen de aquéllos que sólo publican en el país. Conviene también aclarar que la singularidad del caso argentino es de doble factura. No sólo por la inexistencia de incentivos salariales para la publicación mainstream sino también por la convivencia de dos carreras de investigación con sus propios criterios y sistemas de evaluación: la categorización del Programa de Incentivos a Docentes-Investigadores de las universidades nacionales y la carrera de Investigador del CONICET (Beigel, 2015).

\section{El anclaje local de la producción de conocimiento}

Nadie duda que la creencia en la eficacia de los indicadores bibliométricos ha cruzado fronteras espaciales, disciplinares y lingüísticas. Pero exis-

\footnotetext{
2 La Resolución 2249/2014 del Directorio del CONICET de Argentina orienta la clasificación de las publicaciones en tres grupos reconociendo a SciELO en el grupo 1. Sin embargo, la gran cantidad de revistas indexadas en Latindex-Catálogo promovió acuerdos de usos y costumbres que llevaron a las comisiones asesoras a valorar en el grupo 1 también a las publicaciones que estén evaluadas en ese Catálogo, siempre que cumplan con la condición de evaluación externa y ciega. Queda pendiente considerar con igual calidad a REDALYC y la discusión necesaria sobre el carácter internacional de las revistas argentinas evaluadas por CAICYT que también deberían pertenecer al Grupo 1.
} 
ten numerosos estudios empíricos que muestran que la producción científica de las periferias se desarrolla en diversos circuitos transnacionales, regionales, nacionales y locales, lo cual demuestra que estos espacios académicos no están completamente subyugados por la creencia en el Fl o H Index. Lejos de haber desaparecido, la autonomía académica nacional para determinar políticas científicas y criterios de evaluación parece navegar en aguas turbulentas, siempre movilizadas por las injerencias heterónomas, pero sin naufragar completamente.

Observadas empíricamente, vemos que las culturas evaluativas nacionales no reproducen mecánicamente las jerarquías impuestas por los rankings de universidades. La acreditación de instituciones o carreras individuales contempla siempre una serie de instancias intermedias que filtran, clasifican y negocian la "valuación" de criterios globales/necesidades locales. Y los comités de pares o comisiones asesoras operan sobre un marco de referencia que pauta la asignación del mérito (Beigel y Bekerman, 2019). Por otra parte, la publicación en circuitos mainstream desde la periferia no garantiza el reconocimiento académico en todos los terrenos, sino principalmente en el plano nacional. La construcción de un prestigio global ha sido siempre más bien una excepción. Y a nivel institucional puede ser limitado porque muchas universidades promueven el desarrollo de un "habitus" local de circulación. Por lo tanto, en un concurso docente en una universidad no-metropolitana, por ejemplo, un perfil con aquel tipo de publicaciones puede perfectamente perder frente a un candidato/a con publicaciones locales/nacionales.

La dinámica de los circuitos de publicación no anula la existencia de los campos allí donde la política científica y universitaria es dominantemente pública, porque la acreditación de institu- ciones y las carreras académicas siguen siendo pautadas a nivel nacional. La complejidad creciente de la espacialidad académica se verifica al nivel de un campo nacional pero también en las propias instituciones que están atravesadas por diversas formas de circulación y escalas de consagración. Para analizar estos campos científicos segmentados hemos recuperado el concepto de heterogeneidad estructural legado por el latinoamericanismo (Beigel, Gallardo y Bekerman, 2018).

En una universidad dada podremos imaginar que sus estilos de producción dependen de la combinación de varios factores, particularmente las políticas de estímulo a la investigación, las diferencias disciplinares y las culturas evaluativas. Los investigadores/as que publican en revistas de su propia institución (con o sin indexación) pueden alcanzar un reconocimiento local mientras es más difícil que detenten una consagración internacional. Por su parte, los investigadores/as que circulan profusamente en el circuito mainstream y publican en inglés quizás alcancen un rol relevante en consorcios internacionales de investigación colaborativa, pero seguramente capitalizarán esta producción a nivel nacional. En el caso de los investigadores/as de ciencias sociales y humanas es muy común que circulen ampliamente por América Latina logrando eficazmente participar en una agenda regional, ocupando roles de relevancia en asociaciones latinoamericanas o conferencias de CLACSO, por ejemplo. Pero también hay quienes publican en revistas latinoamericanas y, sin embargo, tan sólo capitalizan esta circulación en promociones dentro de organismos nacionales como el CONICET argentino sin lograr ganar un concurso docente en una universidad pública.

Ahora bien, más allá de las estrategias individua- 
les existen estilos institucionales de circulación que se relacionan con la dinámica local pero también con la particular absorción que realizan de las políticas nacionales y oportunidades globales. Hay universidades que promueven únicamente la orientación internacional de sus profesores, mientras otras impulsan ampliamente la extensión (una función que también tiene una larga tradición no sólo en Argentina sino en América Latina) y la participación en la agenda local. En estas últimas, la relación entre la universidad y las organizaciones comunitarias otorga un signo particularmente rico a su vinculación con el medio. Por supuesto, hay universidades "mestizas" y otras de estilo menos definido, lo que depende en buena medida de la síntesis resultante de la interacción entre las políticas científico tecnológicas, las culturas evaluativas y las estrategias individuales. Para conocer empíricamente esta diversidad estamos realizando estudios de caso que nos permitirán poner a prueba los indicadores de circulación que hemos creado y, eventualmente, elaborar una tipología de universidades según sus estilos de circulación (Beigel, 2018).

El objetivo de los indicadores de circulación es dar cuenta de la multiplicidad de formas a través de las cuales es producido el conocimiento científico en las universidades latinoamericanas. La intención de fondo es mover el foco de atención desde el "impacto" medido desde bases de datos como SCOPUS o WoS hacia el análisis local de un universo de estilos de producción. Bajo el paradigma de la circulación proponemos un método de recolección inverso a los rankings universitarios. Es decir, desde abajo hacia arriba, invirtiendo el modelo de trabajo tradicional, para observar empíricamente las instituciones y carre- ras académicas en su medio. Estos indicadores procuran recuperar la multi-escalaridad de la circulación e incluyen en su lente todas las interacciones de nivel local, nacional, regional y global. La metodología en curso en los estudios exploratorios combina abordajes cualitativos (prosopografía, entrevistas y observación participante) y estudios cuantitativos (encuestas y relevamiento de indicadores). Los indicadores de circulación se organizan en 4 dimensiones relacionadas con las funciones de toda universidad: docencia, investigación, vinculación (incluyendo extensión y transferencia) y producción ${ }^{3}$.

\section{Conclusiones}

Las intervenciones impulsadas por la globalización académica no son procesadas por cada institución independientemente, o por los individuos sin mediaciones, sino que están conectadas con las políticas nacionales que operan reforzando tendencias. En algunos países latinoamericanos, la autonomía universitaria sigue siendo una tradición fuerte. En otros, se han concentrado en insertar a su comunidad académica en el circuito mainstream para mejorar la performance de sus universidades en los rankings y aumentar así la cantidad de matrícula/“clientes". Pero, aún en esos contextos, se puede observar la resistencia de una agenda local conducida por investigadores preocupados por vincular el conocimiento con las necesidades sociales y productivas. Buena parte de esta producción se encuentra en la extensión universitaria que es una función presente en universidades de todo el continente. Es hora de revalorizar esos conocimientos y el diálogo de saberes que suponen.

\footnotetext{
${ }^{3}$ En tanto herramienta técnica de observación social, los indicadores de circulación y la tipología deberían colaborar a diseñar/potenciar políticas públicas y definir la asignación estratégica de los recursos no sólo de las instituciones involucradas sino también a los gobiernos provinciales y los organismos decisores del sistema científico-universitario. El Manual de Cuyo que contiene estos indicadores está siendo puesto a prueba en tres universidades y será publicado una vez finalizada la etapa exploratoria.
} 
También subsiste y crece la producción en revistas indexadas en América Latina que poseen una calidad académica probada y profesionalizada. El dinamismo de este circuito regional se debe en gran medida, como hemos visto, a que se construye sobre una plataforma intelectual con una larga historia. Su fortaleza en el tiempo se nutre del carácter dominantemente público de la investigación científica en la región y de su tradición universitaria. Pero es urgente visibilizarla mediante indicadores que mejoren su incidencia en el reconocimiento de carreras académicas y promuevan este circuito en las culturas evaluativas. El debate de la ciencia abierta lanzado en Europa demuestra que los centros están girando su mirada hacia las formas de publicación que en nuestra región llevan décadas de existencia. Y eso está produciendo un renovado interés en los ministerios y agencias científicas de la región que puede promover las condiciones para una metamorfosis de las culturas evaluativas latinoamericanas y así tender un puente en el circuito regional. Por su parte, el Plan S no puede desconocer los efectos nocivos que las políticas de transferencia del costo a los/as autores pueden generar sobre las desigualdades estructurales que operan en el sistema académico mundial actual. Pero además se beneficiaría mucho de un verdadero diálogo con un circuito que hizo del acceso abierto su noble camino hacia la comunicación abierta y colaborativa.

\section{Bibliografía}

Abarzúa Cutroni, A. (2017) "Partículas universales: las misiones científicas de la UNESCO en Argentina (1954-1966)" en CTS: Revista iberoamericana de ciencia, tecnología y sociedad, Vol. 12, №. 36, 2017, págs. 33-60.

Alperin, J.P., y Fischman, G. (Eds.) (2015) Hecho en
Latinoamérica. Acceso abierto, revistas académicas e innovaciones regionales. Buenos Aires: CLACSO.

Alperin, J. P. y Rozemblum, C. (2017) «La reinterpretación de visibilidad y calidad en las nuevas políticas de evaluación de revistas científicas » en Revista Interamericana de Bibliotecología, vol. 40, núm. 3.

Arvanitis, R. et Gaillard, J. (1992) Vers un renoveau des indicateurs de science pour les pays en developpement. Paris: L'Orstom.

Babini, D. (2011). Acceso abierto a la producción científica de América Latina y el Caribe. Identificación de principales instituciones para estrategias de integración regional. Revista Iberoamericana de Ciencia Tecnología y Sociedad, 6, 31-56. https://doi.org/ISSN 1850-0013

Babini, D. (2019) Entrevista realizada por el International Research Council. En https://coun cil.science/current/blog/plan-s-and-open-ac cess-interview-with-dominique-babini

Beigel, Fernanda (Dir.) (2010) Autonomía y Dependencia Académica: Universidad e investigación científica en un circuito periférico. Chile y Argentina (1950-1980), Biblos: Buenos Aires, p.65-88.

Beigel, F. (Ed.) (2013) The politics of academic autonomy in Latin America. Ashgate: London.

Beigel, Fernanda (2014), "Publishing from the Periphery: Structural Heterogeneity and Segmented Circuits. The Evaluation of Scientific Publications for Tenure in Argentina's CONICET". Current Sociology, vol. 62, № 5, pp. 743-765.

Beigel, F. (2015) "Culturas [evaluativas] Alteradas", en Política Universitaria, №2, IEC-CONADU, p.11-21.

Beigel, F (2018) "Las relaciones de poder en la ciencia mundial. Un anti-ranking para conocer la 
ciencia producida en la periferia". Nueva Sociedad, N²74, p.13-28.

Beigel, F.; Gallardo, O. y Bekerman, F. (2018). Institutional expansion and scientific development in the periphery. The structural heterogeneity of Argentina's academic field (1983-2015). Minerva. A Review of Science, Learning and Policy, Enero, disponible en: https://doi.org/10.1007/ s11024017-9340-2

Beigel, F. y Bekerman, F. (2019) Culturas evaluativas en Argentina: Iuces y sombras del Programa de Incentivos a Docentes-Investigadores (1993-2018), CLACSO-CONADU: Buenos Aires. En prensa.

Gareau, F. H. (1988). Another Type of Third World Dependency: the Social Sciences. International Sociology. https://doi. org/10.1177/026858088003002005

GUÉDON, Jean-Claude (2011). El acceso abierto y la división entre ciencia "principal" y "periférica”. Crítica y Emancipación, (6): 135-180.

Heilbron, J., (2002). La bibliométrie, genèse et usages. https://doi.org/10.3917/arss.141.0078

Quesada, F. (2019) "La red de expertos agrícolas de la Fundación Rockefeller en Chile. Entre dinámicas transnacionales y anclajes locales". Workshop: La ciencia y sus públicos. Circulación, apropiación y creación científica en Iberoamérica, siglos XXI y XX. 5 y 6 de septiembre de 2019, Universidad Adolfo Ibáñez, Santiago de Chile.

Salatino, M. (2018). La estructura del espacio latinoamericano de revistas científicas. Tesis de doctorado en ciencias sociales. Universidad Nacional de Cuyo. Disponible en: http://bdigital.uncu.edu.ar/objetos_digitales/10720/salati no-estructuraespaciolatinoamericano-revistascientficas.pdf

Schott, T. (1988). International influence in scien- ce: Beyond center and periphery. Social Science Research, 17(3), 219-238.

Vasen, F. (2018) La 'torre de marfil' como apuesta segura: Políticas científicas y evaluación académica en México. Archivos Analíticos de Políticas Educativas, 26(96). http://dx.doi.org/10.14507/ epaa.v26.3594

Vessuri, H. (1995) "Recent strategies for adding value to scientific journals in Latin America" in Scientometrics, Vol. 34, №1, p. 139-16. 


\section{EL CIRCUITO LATINOAMERICANO EN PERSPECTIVA HISTÓRICA}
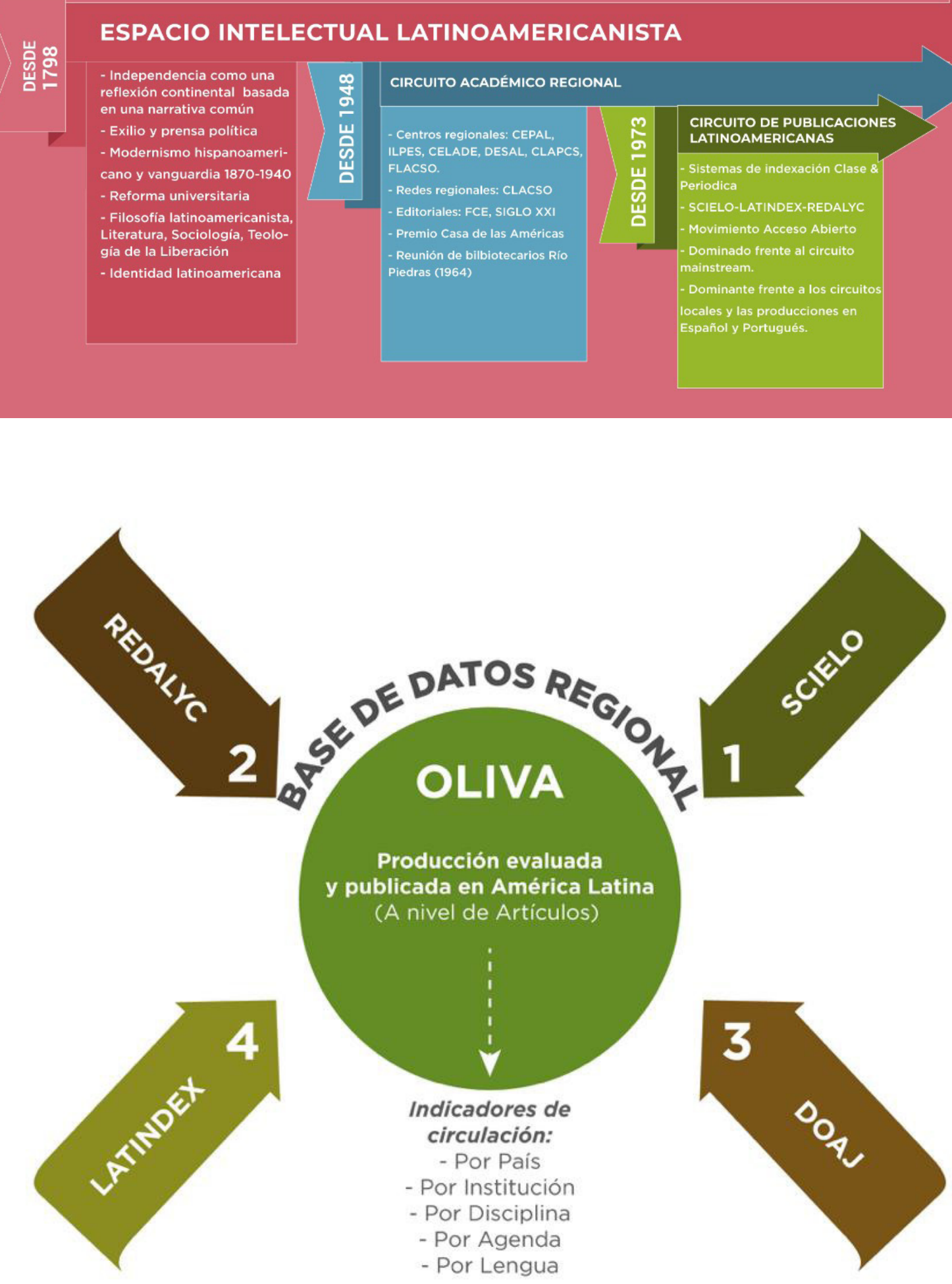


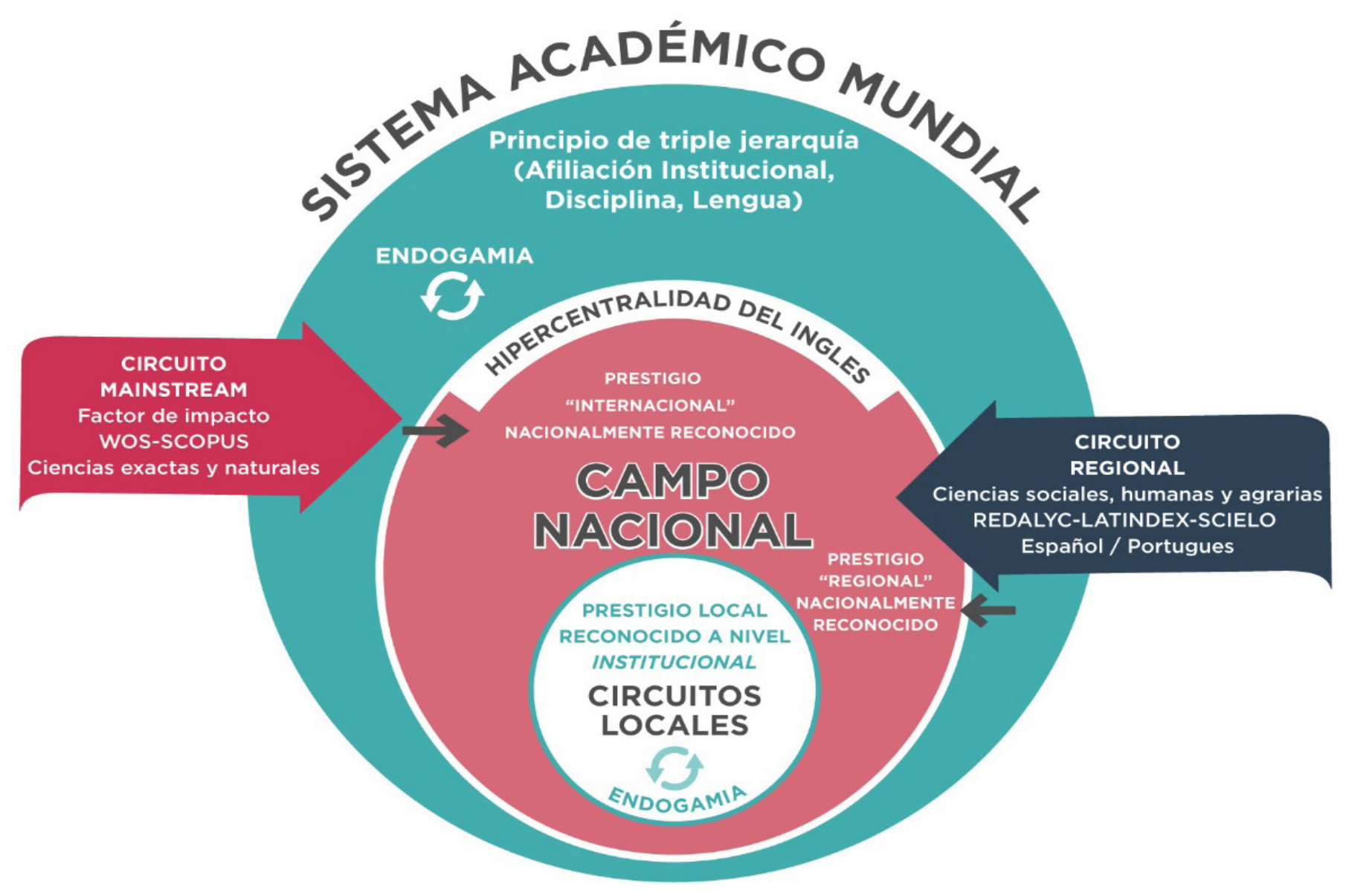

